Review

\title{
Advances in CT Colonography for Colorectal Cancer Screening and Diagnosis
}

\author{
Judy Yee ${ }^{\varpi}$, Stefanie Weinstein, Tara Morgan, Patrick Alore, Rizwan Aslam \\ Dept. of Radiology and Biomedical Imaging, University of California, San Francisco, San Francisco, CA 94121, USA
}

$\triangle$ Corresponding author: Judy Yee, MD. Professor and Vice Chair of Radiology and Biomedical Imaging, University of California, San Francisco, Chief of Radiology, San Francisco VA Medical Center, 4150 Clement Street, San Francisco, CA 94121, 415-750-2120 Judy.yee@radiology.ucsf.edu.

(c) Ivyspring International Publisher. This is an open-access article distributed under the terms of the Creative Commons License (http://creativecommons.org/ licenses/by-nc-nd/3.0/). Reproduction is permitted for personal, noncommercial use, provided that the article is in whole, unmodified, and properly cited.

Received: 2013.01.15; Accepted: 2013.02.07; Published: 2013.03.01

\begin{abstract}
CT colonography (CTC) is a validated colorectal cancer test that provides an additional minimally-invasive screening option which is likely to be preferred by some patients. Important examination prerequisites include adequate colonic cleansing and distention. Tagging of residual material aids in the differentiation of true polyps from stool. Low radiation dose technique should be employed routinely for screening studies. Readers must be skilled in the use of both 2D and 3D interpretation methods.
\end{abstract}

Key words: CT colonography, colorectal cancer

\section{Introduction}

Colorectal cancer (CRC) is the third most common malignancy in the United States with an estimated 143, 460 new cases and 52, 470 deaths in 2012[1] It is also one of the most common malignancies worldwide with an annual global estimate of 1,200,000 new cases and 600,000 deaths from colorectal cancer. [2] The majority of colorectal malignancies are adenocarcinomas and the majority of cases arise from a precursor adenomatous polyp. Thus, this malignancy is ideally suited for screening with the goal being to detect and remove the nidus adenomatous polyp allowing for cancer prevention.CT colonography (CTC), which is also referred to as virtual colonoscopy, was first introduced in 1994 and has become increasingly disseminated worldwide as both a diagnostic test and as a screening tool for colorectal cancer.

Patient compliance rates for colorectal cancer screening continues to be suboptimal in spite of the availability of traditional screening tools such as optical colonoscopy, flexible sigmoidoscopy, double contrast barium enema, fecal occult blood test and fecal immunochemical test. CTC is a validated test that is a fast, less invasive screening test for colorectal carcinoma that provides an option particularly for patients who might otherwise remain unscreened. An overview of CTC examination prerequisites including colonic cleansing and distention and multi-detector $\mathrm{CT}$ technique are discussed. The use of low radiation dose, the main interpretation methods and the latest results of validation trials are included.

\section{Current Status}

CT colonography has been included as a valid option in the joint guideline for colorectal cancer screening of average-risk individuals of the American College of Radiology, the United States Multi-Society Task Force on Colorectal Cancer, and the American Cancer Society[3]. Screening CTC is recommended every five years starting at the age of 50. CTC is being increasingly disseminated for both diagnostic and screening purposes. In the United States national re- 
imbursement for diagnostic CTC is available, although screening CTC is still not reimbursable by the Centers for Medicare and Medicaid Services (CMS). Advantages of CT colonography include minimal invasiveness, a lower risk of complications and increased patient tolerance. A study on patient acceptance and preference comparing CT colonography, optical colonoscopy and double-contrast barium enema demonstrated that patients prefer CTC[4]. In considering imaging tests, the American College of Radiology appropriateness criteria on colorectal cancer screening rates CTC a level of 8 for average-risk individuals, which is higher than the rating for double-contrast barium enema (rating of 7) indicating that CTC is more appropriate as a screening tool for colorectal cancer[5].

Evaluation of more acceptable low volume bowel-cleansing regimens is being performed in order to improve patient adherence and compliance with CTC. These regimens include decreasing the laxative dose and optimizing tagging procedures. Radiation exposure has been decreased with low-dose CT scans. The use of newer techniques such as iterative reconstruction and automatic exposure control will further lower radiation dose to close to background levels. The development of computer aided detection as either a primary or secondary reader is being evaluated to identify more time-efficient and accurate interpretation of CTC.

\section{Validation Trials}

The National CT colonography trial conducted by the American College of Radiology Imaging Network (ACRIN) included 2,531 asymptomatic adults at 15 study sites. Patients in this trial underwent CTC on a minimum 16 row multi-detector CT scanner following standard bowel cleansing and tagging. Colonic distention was achieved with electronic insufflation of carbon dioxide. Readers were all trained radiologists. The reference standard for the study was same day optical colonoscopy. Results included sensitivity, specificity, positive and negative predictive values of $0.90,0.86,0.23$, and 0.99 respectively. Based on the excellent results of this prospective multi-center trial it was suggested that CTC is a valid screening tool for colorectal cancer in an asymptomatic population[6].

CTC was evaluated prospectively in a multi-center study in 937 high-risk patients. Inclusion criteria included a family history of colorectal cancer, personal history of colorectal adenomas, or positive fecal occult blood test. Patients underwent CT colonography followed by same day optical colonoscopy. The sensitivity for the detection of $6-9 \mathrm{~mm}$ and $10 \mathrm{~mm}$ polyps was $85.3 \%$ and $90.8 \%$ respectively, with a specificity of $87.8 \%$. Results confirmed that CT colonography is also a reliable examination in patients at high-risk for CRC[7].

Various CRC screening tests were compared in a study of 307 patients who received fecal immunochemical test and fecal occult blood test prior to CTC and optical colonoscopy after the CTC. Flexible sigmoidoscopy findings were extracted from the optical colonoscopy when considering only the rectosigmoid. A standardized CTC protocol was used for bowel preparation, tagging, colonic distention and CT settings. Sensitivities for the detection of advanced colonic neoplasia for CTC, sigmoidoscopy, fecal immunochemical test and fecal occult blood test when compared to $\mathrm{OC}$ as the reference standard were $96.7 \%, 83.3 \%, 32 \%$ and $20 \%$, respectively. Results demonstrated that CTC performance is comparable to optical colonoscopy and superior to other CRC screening tests[8].

\section{Useful technical tips for CTC perfor- mance}

\section{Regimen selection for Laxative Cleansing}

Patients are required to undergo colonic cleansing on the day before the CT colonography examination similar to patients undergoing optical colonoscopy, although non-cathartic CT colonography techniques are under development and the results of preliminary trials are promising. Patients must also undergo dietary limitation and are not allowed to ingest solids on the day prior to the CT scan. A clear liquid diet is necessary with no dairy products. The laxatives that have been used for colonic cleansing for CTC are classified into two broad categories. The first category is the "dry preparation" laxatives consisting of saline cathartics including magnesium citrate and sodium phosphate. Dry preparation cathartics are preferred for CTC because more of the colon wall is typically revealed when there is less residual fluid in the colon lumen. Saline cathartics are osmolar agents and cause fluid to shift into the small bowel lumen which helps to liquefy stool and promotes evacuation. The second category is the "wet preparation" cathartics typically consisting of large volume polyethylene glycol (PEG) which is commonly used prior to optical colonoscopy and surgery. PEG is an electrolye lavage preparation that functions as an osmolar agent that increases the water content of stool and induces elimination.

\section{Magnesium citrate}

Magnesium citrate is a well-tolerated laxative used for CTC. It is available as a pre-mixed solution 
and the patient is instructed to ingest 10 ounces in the late afternoon or early evening on the day prior to the CT scan. Alternatively magnesium citrate is available in a powder form that the patient dissolves in 8 ounces of clear liquid prior to ingestion. It typically takes approximately one hour for the magnesium citrate to take effect. Four $5 \mathrm{mg}$ bisacodyl tablets are taken about three hours after the magnesium citrate and will promote additional catharsis 6-12 hours after use. A $10 \mathrm{mg}$ bisacodyl suppository is administered the morning of the CT examination which promotes final evacuation of the distal colon about 15 to 30 minutes following insertion into the rectal vault. Clear instruction should be given to the patient to have access to a restroom during the entire preparation period. It is suggested that patients request scheduling of their CTC examination in the morning which allows for improved patient comfort and easier patient adherence to required dietary restrictions.

Magnesium citrate is less likely to result in clinically significant electrolyte imbalances compared to sodium phosphate although fluid intake should still be maintained to prevent dehydration. In a comparison study of double dose magnesium citrate to single-dose sodium phosphate, results showed excellent cleansing for both with no significant difference in amounts of residual stool and fluid[9]. In this study use of the magnesium citrate preparation resulted in a statistically significant more optimum higher residual fluid attenuation value with a mean of 790 Hounsfield Unit versus 978 Hounsfield Unit with the use of sodium phosphate. Patients with renal insufficiency or who may be at risk for phosphate nephropathy or electrolyte disturbances should receive magnesium citrate rather than sodium phosphate.

\section{Sodium phosphate}

Another saline cathartic that has been used for CTC is sodium phosphate. This is typically ingested as a single dose of 1.5 ounces mixed with 4 ounces of water and then followed with an additional 8 ounces of water. Similar to magnesium citrate, it should be ingested in the late afternoon or early evening on the day before the CT scan. It takes about one hour for the laxative effect to occur and patients are advised to remain close to a restroom during this preparation period. Similar to the magnesium citrate preparation, four $5 \mathrm{mg}$ bisacodyl tablets are taken about three hours after the sodium phosphate. A $10 \mathrm{mg}$ bisacodyl suppository is administered the morning of the CTC. Although a double dose of sodium phosphate consisting of 3 ounces has been used for CTC in the past, it is currently not recommended because of the higher frequency of possible electrolyte abnormalities such as hypernatremia, hyperphosphatemia, hypocalcemia and hypokalemia. Acute phosphate nephropathy is another uncommon complication. The Food and Drug Administration (FDA) has issued a warning for the use of 3 ounces of sodium phosphate[10]. Contraindications to the use of sodium phosphate include electrolyte disturbances, renal failure, congestive heart failure, and ascites. Patients taking angiotensin converting enzyme (ACE) inhibitors or who are prone to dehydration should not receive sodium phosphate. A study comparing the use of single dose versus two doses of sodium phosphate found that both were equally effective for colonic cleansing for CTC[11].

\section{Polyethylene glycol}

Polyethylene glycol is a synthetic insoluble polymer that functions as an osmotic laxative and is commonly used as a prescription large volume liquid bowel cleansing agent prior to colonoscopy and surgery. Currently it is also available over the counter in a powder form to treat constipation. Various polyethylene glycol solutions are available in combination with different electrolytes that may include sodium chloride, potassium chloride, sodium bicarbonate, sodium sulfate, ascorbic acid, or sodium ascorbate. PEG does not typically induce large fluid shifts and therefore may be preferred in elderly patients. Although serious complications such as electrolyte abnormalities, anaphylaxis, vomiting, and aspiration have been reported with the use of PEG they are uncommon. When used for bowel cleansing prior to CTC, four liters of PEG is prescribed for ingestion within a 2 to 3 hour interval on the evening before the day of the CT scan. Bisacodyl tablets and a suppository are administered similar to the saline cathartics.

Large volume polyethylene glycol (four liters) is not the preferred bowel cleansing agent for CTC. Although PEG is effective in softening and evacuating residual stool, it leaves behind sizeable fluid pools. Large amounts of residual fluid may result in heterogeneous tagging of residual material. A study evaluating amounts of residual fluid in patients receiving sodium phosphate versus polyethylene glycol prior to CTC found that there was significantly less residual fluid with the use of the sodium phosphate[12]. In the American College of Radiology Imaging Network (ACRIN) National CT Colonography study, patients received three different bowel cleansing agents including polyethylene glycol $(\mathrm{n}=1403)$, sodium phosphate $(n=1020)$ and magnesium citrate $(n=102)$. Results showed that there was no difference in polyp detection rates irrespective of the bowel cleansing agent used[13]. Another disadvantage of polyethylene glycol electrolyte solution is difficulty with patient 
compliance due to abdominal discomfort, nausea, and bloating. The solution also has a salty taste which may be improved with various flavored versions that are commercially available.

\section{Others}

New regimens employing less rigorous bowel cleansing regimens are being evaluated for CTC in order to improve patient tolerance. Four different protocols were studied in a trial evaluating bisacodyl[14]. Three regimens included increasing amounts of bisacodyl and one protocol included bisacodyl in combination with magnesium citrate. A two-day low-fiber diet was administered and tagging was performed using diatrizoate meglumine and barium. The study found no significant difference in image quality or tagging quality even with the larger laxative amounts. Lower dose cleansing regimens resulted in improved patient acceptance rates. In another study half volume polyethylene glycol (two liters) was compared to magnesium citrate in patients undergoing CTC[15]. Adequate cleansing with better tagging and shorter interpretation times were found in the patients who received the reduced volume PEG.

\section{Tagging}

Tagging of residual material in the colon has been increasingly employed as a method that can help to improve the ability to detect true polyps and to decrease the number of false positives identified on CTC. Oral contrast agents are used to label residual solid stool and fluid so that they are higher density and more easily distinguished from the homogeneous soft tissue density of polyps and cancers. Typically doses of barium sulfate and/or iodinated contrast material are ingested at specified times starting one to two days prior to the CTC.

High density and low density barium products have been shown to be effective for tagging residual material for CTC[16]. Iodinated contrast agents are hyperosmolar and cause fluid shifts which results in an additional cathartic effect. Patients participating in the ACRIN National CTC Trial ingested two tagging agents including $40 \mathrm{~mL}$ of high density $40 \%$ weight/volume barium sulfate given in 3 aliquots during the morning before the CT scan and $60 \mathrm{~mL}$ of iodinated contrast given the evening before the CT scan. Bowel cleansing was performed using PEG, magnesium citrate or sodium phosphate and bisacodyl tablets[17]. Another tagging protocol consists of $250 \mathrm{~mL}$ of low density $2 \%$ weight/volume barium sulfate and $60 \mathrm{~mL}$ of sodium diatrizoate/diatrizoate meglumine also administered on the day before the CTC. This is typically given 2-3 hours following bowel cleansing with either PEG, magnesium citrate, or sodium phosphate and bisacodyl tablets[18]. Iodine-based regimens have been found to result in more homogeneous tagging of residual material compared with barium tagging for non-laxative or minimum-laxative CT colonography[19]. CTC without cathartic preparation was performed in 1,920 average risk patients who ingested $60 \mathrm{~mL}$ of iodinated contrast[20]. The per-patient positive predictive value (PPV) was $92.8 \%$ with similarly high per-polyp PPV for polyps $6 \mathrm{~mm}$ and larger.

Patients may be referred for same day CTC following incomplete optical colonoscopy. Tagging can be performed with an iodinated contrast agent following incomplete colonoscopy using a shortened interval protocol. In a study of 144 patients who ingested $20-30 \mathrm{~mL}$ of iodinated contrast 2 hours prior to CT colonography and following incomplete colonosocopy, results showed that over $70 \%$ of patients had adequate tagging with contrast reaching the distal colon[21].

\section{Gaseous Distention}

Adequate distention of the colonic lumen is essential to allow for detection of lesions on two- and three-dimensional images. Poorly distended areas of the colon can simulate annular carcinomas and also cause for missed polyps. A small caliber flexible rectal tube (typically 18 French or 20 French gauge) is placed in the rectal vault to allow for administration of either room air or carbon dioxide. Some catheters have an inflatable balloon near the tip. The scout image is used to assure that the entire colon is distended from rectum to cecum. Current standard of care requires the patient to be scanned in supine and prone positions in order to shift fluid pools and stool as well as to achieve improved distention, particularly of the sigmoid colon[22]. A limited repeat scan may be obtained with the patient in a decubitus position of a poorly distended segment.

Gaseous distention of the colon is typically achieved with either room air or carbon dioxide administered in a retrograde fashion. CTC was originally performed using manual administration of room air per rectum. However, since there is no absorption of room air across the colon wall this can lead to significant abdominal pain and distention during and after the CTC examination. Carbon dioxide (CO2) is commonly used as an alternative to room air due to its improved patient tolerance. Carbon dioxide has higher lipid solubility resulting in net absorption across the bowel wall and allowing for more rapid deflation of the bowel wall after the CT exam. A mechanical device is used to easily and safely administer 
the CO2. The electronic insufflator allows for careful monitoring of colonic pressure which enables the ability to obtain optimal distention of the colon safely and has been found to provide better distension compared to manual insufflation[23]. $\mathrm{CO} 2$ has been found to be better tolerated than room air because of decreased intra- and post-procedural abdominal pain and discomfort[24]. Automated carbon dioxide insufflation has also been found to be effective for colon distention on CTC in colorectal cancer patients with severe luminal narrowing[25].

The risk of colonic perforation due to CTC is extremely low. An analysis of over 21,000 CTC examinations found a total perforation rate of $0.009 \%$, symptomatic perforation rate of $0.005 \%$ and overall complication rate of $0.02 \%$ [26]. Many perforations observed on CTC occur in patients who are asymptomatic with small amounts of extraluminal gas identified on CT scan that likely would not have been detected using the other colon tests. Studies have found symptomatic perforation rates for CTC to range between $0.005 \%$ and $0.03 \%$. The colonic perforation rate due to optical colonoscopy is at least 10 to 20 times higher and ranges from 0.1 to $0.2 \%$ [27].

\section{Spasmolytic Agents}

A spasmolytic agent is not routinely used for CTC in the United States. Although glucagon has been used as an anti-spasmodic agent for barium enema examinations and in the original development of CTC, it has not been found to significantly improve bowel distention or polyp detection on CTC[28]. Additionally use of glucagon is associated with increased cost and the potential for side effects such as nausea and vomiting as well as uncommon allergic reactions such as hypotension and respiratory distress. Glucagon may be administered in specific patients with significant abdominal discomfort or if there is persistent colonic spasm.

Hyoscine butylbromide (Buscopan) is used as an anti-spasmodic agent in Europe and other countries, although it is not approved for use in the United States. Hyoscine butylbromide is an anticholinergic drug that decreases muscular tone of the bowel and has been found to improve bowel distention. In a study comparing hyoscine butylbromide, glucagon and no anti-spasmodic agent, results showed that there was significantly larger colonic volume when comparing hysocine butylbromide to no agent, but no significant difference when comparing glucagon to no agent[29]. Side effects of hyoscine butylbromide include tachycardia, dry mouth, nausea and hypotension. It is contraindicated in patients with prostatism, tachycardia, cardiac failure, bowel obstruction, my- asthenia gravis, and glaucoma[30].

\section{Low Dose CTC Technique}

State-of-the-art CTC is performed on a multidetector CT (MDCT) scanner with a detector row thickness between 0.5 to $0.625 \mathrm{~mm}$. MDCT scanners allow scanning of the abdomen and pelvis with thinner slices, shorter scan times of less than 10 seconds per position, and lower radiation dose. Thin sections help to eliminate partial volume averaging and improve image quality of the sagittal and coronal multiplanar reformatted views as well as the three-dimensional endoluminal images. Recommendations according to the American College of Radiology Practice Guideline for the performance of CT colonography include MDCT scanner parameters of a minimum of 4 detector rows, $3 \mathrm{~mm}$ or less slice thickness and $2 \mathrm{~mm}$ or less reconstruction interval[16]. A sample 64 row multidetector CTC protocol includes effective $\mathrm{mAs}$ of 50 or less, $\mathrm{kVp}$ of 120 and reconstructions of $1-1.25 \mathrm{~mm}$. Thicker reconstructions of 3 to $5 \mathrm{~mm}$ are used for evaluation of extracolonic findings.

The use of CTC as a screening test for colorectal cancer suggests that it is performed at recommended repeated interval. This highlights the importance of using a low radiation dose screening CTC protocol. Tube current is one of the main CT parameters determining image noise that may be adjusted to reduce radiation dose. The detection of polyps on CTC depends on the inherent high contrast between intraluminal gas and the soft tissue density of the colon wall allowing for a significant decrease in the tube current. An initial analysis of 34 sites determined that the median effective dose for screening CTC was $5.7 \mathrm{mSv}$ compared with $9.1 \mathrm{mSv}$ for standard CT of the abdomen and pelvis[31]. Estimated radiation dose to different organs from supine and prone CTC have been calculated[32]. These doses are then applied to organand dose-specific radiation cancer risk estimates to determine the excess cancer risk due to CTC. Results show an estimated $5 \mathrm{mSv}$ dose for screening CTC which is associated with an absolute small lifetime cancer risk of $0.14 \%$ per CTC at age 50 and $0.07 \%$ per CTC at age 70. This is a small risk that may be further reduced by factors of 5 or 10 using optimized CTC protocols. In a study comparing 64 row multi-detector row CTC and double-contrast barium enemas (DCBE), the effective radiation dose from CTC was found to be about half that of DCBE[33]. The benefit to risk ratio of cancers prevented to cancers induced due to screening CTC performed every five years from age 50 to age 80 was calculated using three microsimulation models[34]. The mean effective dose used for this study was $7 \mathrm{mSv}$ for males and $8 \mathrm{mSv}$ for females. 
Results showed that the benefit to risk ratio was very high ranging from 24:1 to $35: 1$ and that the benefits of CTC screening clearly outweigh the risks.

Various techniques are being employed to continue to decrease the radiation dose of CTC. Automatic exposure control (AEC) continuously adjusts the tube current according to the patient's volume and can significantly reduce radiation exposure particularly in thin patients. Radiation exposure may be decreased by as much as 20 to $35 \%$ for abdominal CT scans using AEC[35]. Another technique that has been used to decrease radiation dose is iterative reconstruction which is employed in combination with traditional filtered back projection (FBP) to post-process CT data. Iterative reconstruction technique can process low tube current CT data in a manner that reduces noise and preserves image quality. A pilot study showed the feasibility of preserving image quality during CTC using decreased radiation dose with iterative reconstruction[36]. It was found that the radiation dose during CTC can be reduced 50\% without significantly affecting image quality when iterative reconstruction is used.

\section{Interpretation Methods}

The interpretation of CT colonography requires focused interactive training using specific computer software that allows dynamic viewing of two dimensional axial images, multi-planar reformats (MPR) and three-dimensional renderings. Readers may use either 2D axial images or 3D renderings for primary interpretation of CTC, with the alternate method reserved for problem solving specific questions related to a potential lesion. Current emphasis is for readers to develop skills for CTC interpretation using both two and three dimensional images in order to optimize lesion detection and to decrease interpretation times. Proficient utilization of these techniques, gained by appropriate training, has been shown to correlate with polyp detection sensitivity. The sensitivity for detection of polyps $6 \mathrm{~mm}$ or larger during the training period was found to be the sole independent predictor for subsequent sensitivity for similar lesion detection[37].

Primary 2D interpretation is performed by scrolling through magnified axial images of the colon obtained in supine and prone positions. CT window settings should be set to maximize detection of intraluminal lesions; high contrast colon window settings should approximate a window width of 1,500 and level of -300 . Sessile polyps have round or ovoid morphology and are of soft tissue density. They remain fixed in location on the colon wall in both the supine and prone images. [FIGURE 1] Stool can be differentiated from polyps since it is typically of mixed density and shifts location when the patient changes position. Pedunculated polyps can shift in location when the patient moves from supine to prone positions, but the stalk is typically easily identified on 2D and 3D images. [FIGURE 2] Multiplanar reformats and 3D images are useful for evaluating lesion morphology and for confirming polyps. Primary 2D interpretation allows for rapid assessment of lesion density and homogeneity and has been shown to have overall relatively shorter interpretation times compared to primary 3D interpretation[6].

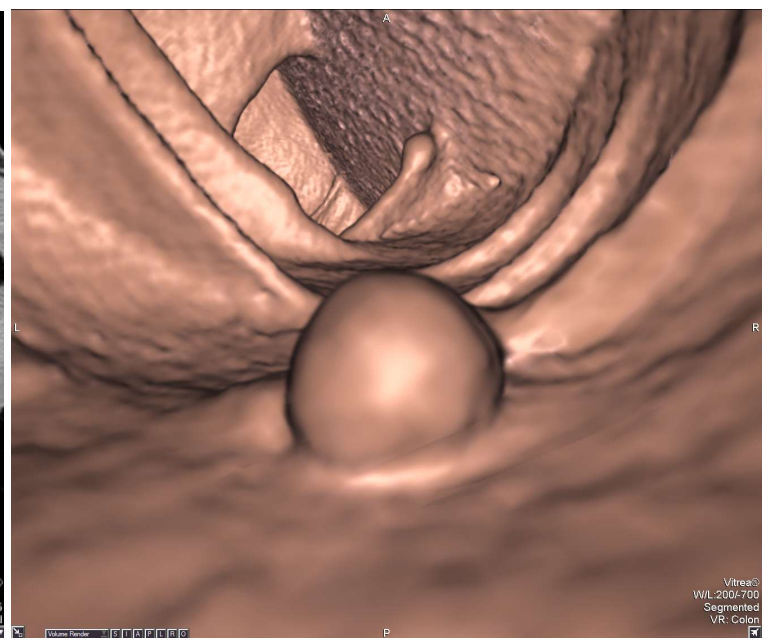

Figure I - Sessile Polyp. Axial view (A) shows a sessile polyp in the ascending colon. The 3D endoluminal view (B) reveals a typical spherical appearance of a sessile polyp. 

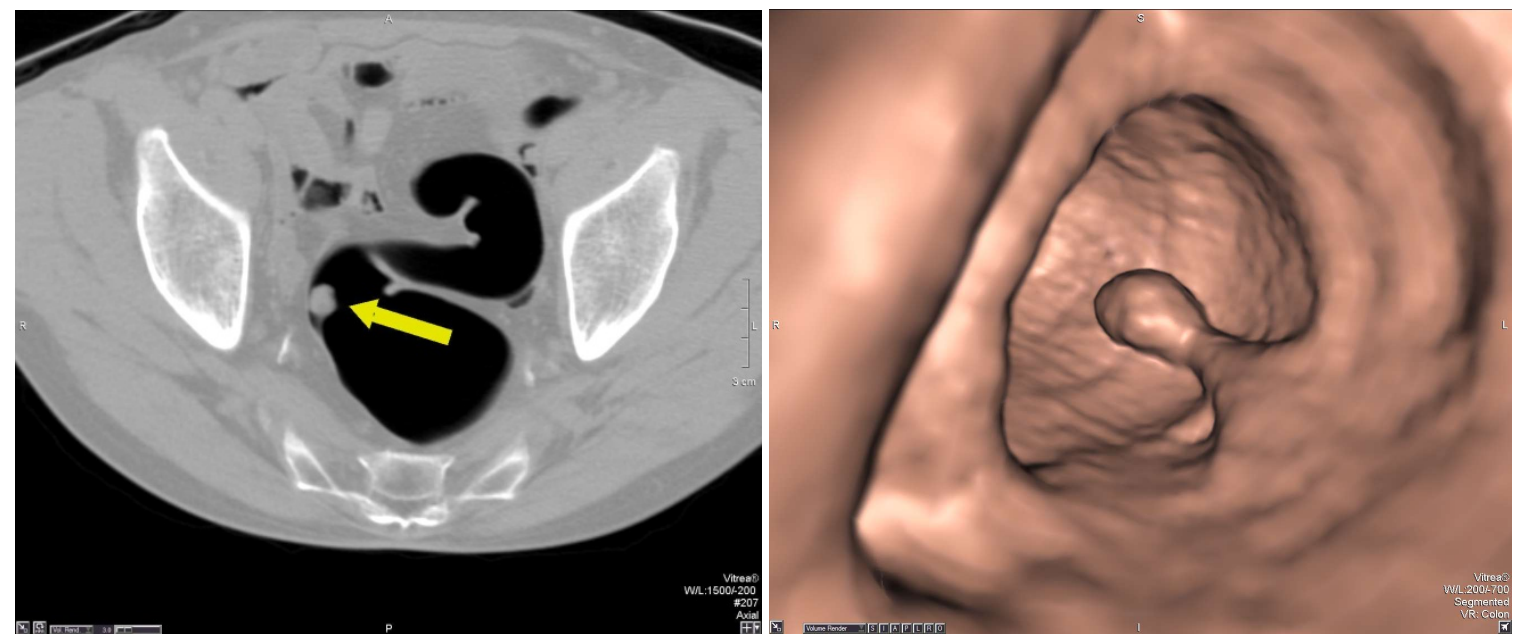

Figure 2 - Pedunculated Polyp - Axial view (A) shows a large pedunculated polyp with a short stalk in the sigmoid colon. The 3D endoluminal view (B) shows the pedunculated polyp arising from a haustral fold and projecting into the lumen.

Primary 3D interpretation is performed by endoluminal navigation through the entire colon lumen along a computer-generated center line. Anterograde and retrograde navigations in both supine and prone positions are required in order to assure complete assessment of the colon wall. Evaluation during both retrograde and anterograde navigations allows for visualization of both sides of colonic folds. Similarly, layering fluid or residual stool could obscure polyps when viewing 3D navigations in only one position. When a potential lesion is identified on the $3 \mathrm{D}$ view, the reader places a bookmark near the lesion and then evaluates the lesion density using the axial or multiplanar views. Lesion mobility should then be assessed by comparing the lesion location and orientation from supine to prone positions. Additional CTC software algorithms are available to improve discrimination of true polyps from pitfalls. A color density map placed on the $3 \mathrm{D}$ endoluminal view aids in differentiating densely tagged stool from the soft tissue density of polyps without having to refer back to the 2D display. Another algorithm paints the viewed surfaces of the colon on the $3 \mathrm{D}$ endoluminal view a different color and then presents a list of patches of wall that have not been previously viewed so that the reader can rapidly perform a complete review of all surfaces.

The primary $3 \mathrm{D}$ interpretation technique may be easier for some inexperienced readers to learn. Additionally polyps on or near colon folds are often seen better on 3D views. Interpretation times for primary $3 \mathrm{D}$ reads tend to be longer than for primary $2 \mathrm{D}$ reads. Comparison of primary 2D versus primary 3D interpretation methods was performed as a part of the ACRIN CTC trial[38]. The sensitivity and specificity for primary $2 \mathrm{D}$ readers was 0.84 and 0.86 respectively for polyps $10 \mathrm{~mm}$ or larger. Readers who employed both 2D and 3D techniques demonstrated a sensitivity and specificity of 0.84 and 0.83 respectively. The sensitivity and specificity of primary 3D readers was 0.76 and 0.82 respectively. There was no statistically significant difference in performance between any of these groups.

Various CT colonography techniques are being evaluated in an attempt to improve accuracy and decrease reading times. The virtual dissection view is a novel display in which the tubular shaped colon is bisected through its long axis and then opened and flattened for an internal display. The dissection view allows visualization of larger portions of the colon wall simultaneously and may help to shorten interpretation times. However, there may be distortion of normal folds and of lesions with the flattening technique so readers must be specifically trained to interpret these views.

Electronic cleansing is a post-processing technique involving application of computer algorithms to subtract intra-colonic high-density tagged material. Residual material in the colon is tagged using ingested barium and/or iodinated contrast administered at specified time periods on the day prior to the CTC. Following electronic cleansing and removal of the tagged stool and fluid, soft tissue polyps and cancers are better visualized. The need for laxative cleansing of the colon may potentially be reduced or eliminated with optimum tagging of residual material combined with electronic cleansing. The effect of electronic subtraction is limited in the presence of partial volume artifacts and heterogeneous tagging of stool. Another potential pitfall of electronic cleansing is "over-subtraction," where parts of soft tissue den- 
sity polyps are removed along with tagged material causing incorrect sizing or false negatives. A study of 114 patients undergoing tagged, non-cathartic CT colonography showed that stool subtraction increased sensitivity for the detection of polyps larger than $1 \mathrm{~cm}$ and polyps between 6 to $9 \mathrm{~mm}$, compared to non-subtracted images[39]. A prospective evaluation of 605 asymptomatic patients evaluating the diagnostic accuracy of laxative-free CTC found that the per-patient sensitivity for the detection of adenomas $10 \mathrm{~mm}$ or larger, $8 \mathrm{~mm}$ or larger and those $6 \mathrm{~mm}$ or larger were $91 \%, 70 \%$, and $59 \%$ respectively[40]. A low osmolar, nonionic iodinated contrast agent was used for tagging.

Computer aided detection (CAD) for CT colonography uses computer algorithms based on polyp features such as shape to identify potential lesions. These computerized methods may be used to improve sensitivity for polyp detection particularly in novice readers and to reduce inter-reader variability. CAD may employed as a primary reader to identify possible polyps and cancers prior to the radiologist's interpretation or as a secondary check after the initial unassisted interpretation by the radiologist in order to confirm findings and identify any additional missed lesions. CAD may also be used as a concurrent reader, in which a radiologist reads the CTC images while assisted by CAD with potential lesions identified as targets by the software. CAD is most commonly used as a secondary reader. A study comparing CTC interpretation using $\mathrm{CAD}$ as a secondary versus concurrent reader found that CAD functioning as a secondary reader significantly improved reader sensitivity but use of CAD concurrently had no effect[41]. Neither concurrent nor secondary CAD improved the specificity of CTC. Increased sensitivity for polyp detection using CAD must be offset by adequate specificity so that false positives are minimized and interpretation times are acceptable. A study comparing CAD applied to both tagged and untagged CTC examinations found that there were no significant performance differences with similar sensitivity of about $90 \%$ for polyps $6 \mathrm{~mm}$ or larger[42]. Detection rates were demonstrated to be comparable to human readers at a relatively low false-positive rate of about 5 per patient dataset.

The CT colonography Reporting and Data System (C-RADS) was developed to promote more consistent and clear communication of CT colonography results[43]. Lesion descriptors including size, morphology, segmental location and density are recommended to assist in standardizing CTC reports. The classification system consists of categories ranging from inadequate study $(\mathrm{C} 0)$ to likely malignant co- lonic mass (C4).

\section{Extracolonic Findings}

Screening CT colonography is performed as a non-contrast CT scan of the abdomen and pelvis. CTC is inherently able to detect incidental lesions external to the colon in contrast to optical colonoscopy and the other colorectal cancer screening tools. The majority of these extracolonic findings are benign and of no clinical significance. However, additional workup of extracolonic findings may lead to increased health care costs, patient anxiety, and potentially patient morbidity. Advantages include identification of clinically significant lesions such as abdominal aortic aneurysms and other malignancies at an earlier stage which can have the benefit of decreased patient morbidity and mortality as well as lower long-term health care costs[44]. The detection of extracolonic abnormalities may be limited with lower dose CT techniques due to increased noise on images. However, new iterative reconstruction techniques have been shown to improve the image quality of these low dose scans[45]. A relatively larger number of significant extracolonic findings have been shown to be identified with the use of intravenous contrast.

Extracolonic lesions are detected in a large percentage of patients undergoing screening CTC. Extracolonic findings are particularly high among senior patient groups, when compared to younger patient groups [46]. However, the percentage of patients, across age and gender, with extracolonic findings that are clinically significant is relatively quite low[47]. Due to the relatively low incidence of polyps and colorectal cancer in the asymptomatic population, the identification of additional clinically important extracolonic lesions can greatly increase the utility and potentially cost-effectiveness of screening CTC. A meta-analysis of approximately 3,500 patients undergoing CTC found extracolonic lesions in $40 \%$ of patients with $14 \%$ undergoing further testing and $0.8 \%$ requiring immediate treatment. Aortic aneurysms and extracolonic malignancies were found in $0.9 \%$ and $2.7 \%$ of the patients, respectively[48]. A retrospective study of 2,277 patients who underwent screening CTC found extracolonic findings in $46 \%$ of patients, with $11 \%$ classified as clinically significant by the interpreting radiologist[49]. A study of greater than 10,000 asymptomatic patients detected unsuspected cancer in $0.56 \%$ of CTC examinations; invasive colorectal cancer in $0.21 \%$ and extracolonic cancer in $0.35 \%$ [50]. Investigation of the application of CTC for non-colorectal cancerous conditions demonstrated that the detection of both colonic and extracolonic findings on CTC reduced the indications for colon- 
oscopy among the study group[51].

An extracolonic classification system for CTC has been developed. Categories range from a limited exam (E0) to an exam with potentially important extracolonic findings (E4)[43]. Guidance for the management of incidental findings on abdominal CT, including low-dose unenhanced $\mathrm{CT}$, is provided in a white paper by the ACR Incidental Findings committee[52].

\section{Competing Interests}

The authors have declared that no competing interest exists.

\section{References}

1. Siegel R, Naishadham D, Jemal A. Cancer statistics, 2012. CA Cancer J Clin. 2012; 62:10-29.

2. Jemal A, Bray F, Center MM, Ferlay J, Ward E, Forman D. Global cancer statistics. CA Cancer Clin 2011;61:69-90.

3. Levin B, Lieberman DA, McFarland B, et al. Screening and Surveillance for the Early Detection of Colorectal Cancer and Adenomatous Polyps, 2008: A Joint Guideline from the American Cancer Society, the US Multi-Society Task Force on Colorectal Cancer, and the American College of Radiology. CA Cancer J Clin 2008;58:130-160.

4. Gluecker TM, Johnson CD, Harmsen WS, et al. Colorectal cancer screening with CT colonography, colonoscopy, and double-contrast barium enema examination: prospective assessment of patient perceptions and preferences. Radiology 2003;227:378-384.

5. Yee J, Rosen MP, Blake MA, Baker ME, et al. ACR Appropriateness Criteria on colorectal cancer screening. J Am Coll Radiol 2010;17:670-8.

6. Johnson CD, Chen M-H, Toledano AY, et al. Accuracy of CT Colonography for detection of large adenomas and cancers. N Engl J Med 2008;359:1207-17.

7. Regge D, Laudi C, Galatola G, et al. Diagnostic accuracy of computed tomographic colonography for the detection of advanced neoplasia in individuals at increased risk of colorectal cancer. JAMA 2009;301:2453-61.

8. Graser A, Stieber P, Nagel D et al. Comparison of CT colonography, colonoscopy, sigmoidoscopy and faecal occult blood tests for the detection of advanced adenoma in an average risk population. Gut 2009;58:241-248.

9. Borden ZS, Pickhardt PJ, Kim DH, et al. Bowel Preparation for CT Colonography: Blinded Comparison of Magnesium Citrate and Sodium Phosphate for Catharsis. Radiology 2010; 254:138-144.

10. United States Food and Drug Administration Website. Oral sodium phosphate (OSP) products for bowel cleansing (marketed as Visicol and Osmo- Prep and oral sodium phosphate products available without a prescription). FDA alert December 11, 2008

11. Kim DH, Pickhardt PJ, Hinshaw JL, et al. Prospective blinded trial comparing $45 \mathrm{~mL}$ and $90 \mathrm{~mL}$ doses of oral sodium phosphate for bowel preparation before computed tomographic colonography. J Compu Assist Tomogr 2007;31:53-8.

12. Macari M, Lavelle M, Pedrosa I, Milano A, Dicker M, Megibow AJ, Xue $X$. Effect of differenct bowel preparations on residual fluid at CT colonography. Radiology 2001;218:274-277.

13. Hara AK, Kuo MD, Blevins $\mathrm{M}$, et al. National CT colonography trial (ACRIN 6664): comparison of three full-laxative bowel preparations in more than 2500 average-risk patients. AJR Am J Roentgenol. 2011;196:1076-82

14. Jensch S, de Vries AH, Pot D, et al. Image quality and patient acceptance of four regimens with different amounts of mild laxatives for CT colonography. AJR Am J Roentgenol 2008;191:158-67.

15. Keedy AW, Yee J, Aslam R, Weinstein S, Landeras LA, Shah JN, McQuaid KR, Yeh BM, Reduced Cathartic Bowel Preparation for CT Colonography: Prospective Comparison of 2-L Polyethylene Glycol and Magnesium Citrate. Radiology in press, 2011.

16. American College of Radiology. ACR practice guideline for the performance of computed tomography (CT) colonography in adults. 2009 http://www.acr.org/SecondaryMainMenuCategories/quality_safety/guidelines/d $\mathrm{x} /$ gastro/ct_colonography.aspx. Accessed July 1, 2012

17. Johnson $\mathrm{CD}$, Chen M-H, Toledano AY, et al. Accuracy of CT Colonography for detection of large adenomas and cancers. N Engl J Med 2008;359:1207-17.

18. Kim DH, Pickhardt PJ, Taylor AJ, et al. CT Colonography versus colonoscopy for the detection of advanced neoplasia. N Engl J Med 2007;357:1403-1412.
19. Lefere PA, Gryspeerdt SS, Dewyspelaere J, et al. Dietary fecal tagging as a cleansing method before CT colonography: initial results polyp detection and patient acceptance. Radiology 2002;224:393-403.

20. Zueco Zueco C, Sobrido Sampedro C, Corroto JD, et al. CT colonography without cathartic preparation: positive predictive value and patient experience in clinical practice, Eur Radiol. 2012; 22:1195-204.

21. Chang KJ, Rekhi SS, Anderson SW, Soto JA. Fluid Tagging for CT Colonography: Effectiveness of a 2-Hour Iodinated Oral Preparation After Incomplete Optical Colonoscopy. J Comput Assist Tomogr 2011;35:91-95.

22. Yee J, Kumar NN, Hung RK, et al. Comparison of supine and prone scanning separately and in combination at CT colonography. Radiology 2003;226:653-661.

23. Burling D, Taylor SA, Halligan S, et al. Automated insufflation of carbon dioxide for MDCT colonography: distension and patient experience compared with manual insufflation. AJR 2006; 186:96-103.

24. Shinners TJ, Pickhardt PJ, Thaylor AJ, Jones AJ, Olsen CH. Patient-controlled room air insufflation versus automated carbon dioxide delivery for CT colonography. AJR Am J Roentgenol 2006;186, 1491-1496.

25. Kim SY, Park SH, Choi EK, et al. Automated carbon dioxide insufflation for CT colonography: effectiveness of colonic distention in cancer patients with severe luminal narrowing. AJR Am J Roentgenol 2008;190:698-706.

26. Pickhardt PJ. Incidence of colonic perforation at CT colonography: review of existing data and implications for screening of asymptomatic adults. Radiology 2006;239:313-6.

27. de Gonzalez AB, Kim KP, Yee J. CT Colonography: Perforation Rates and Potential Radiation Risks. Gastrointest Endoscopy Clinic N Am 2010;20:279-291.

28. Yee J, Hung RK, Akerkar GA, et al. The usefulness of glucagon hydrochloride for colonic distention in CT colonography. AJR Am J Roentgenol 1999;173:1-4.

29. Rogalla P, Lembcke A, Ruckert JC, et al. Spasmolysis at CT colonography: butyl scopolamine versus glucagon. Radiology 2005;236:184-8.

30. Tytgat GN. Hyoscine butylbromide- a review on its parenteral use in acute abdominal spasm and as an aid in abdominal diagnostic and therapeutic procedures. Curr Med Res Opin 2008; 24:3159-3173.

31. Liedenbaum MH, Venema HW, Stocker J. Radiation dose in CT colonography-trends in time and differences between daily practice and screening protocols. Eur Radiology 2007;17:2616-21.

32. Brenner DJ and Georgsson MA. Mass screening with CT colonography:should the radiation exposure be of concern? Gastroenterology 2005;129:328-337.

33. Neri E, Faggioni L, Cerri F, Turini F, Angeli S, Cini L, Perrone F, Paolicchi F, Bartolozzi C. CT colonography versus double-contrast barium enema for screening of colorectal cancer: comparison of radiation burden. Abdom Imaging 2010;35:596-601.

34. de González AB, Kim KP, Knudsen AB, et al. Radiation-related cancer risks from CT colonography screening: a risk-benefit analysis. AJR Am J Roentgenol 2011;196:816-23.

35. Singh S, Kalra MK, Thrall JH, Mahesh M. Automatic Exposure Control in CT: Applications and Limitations. J Am Coll Radiol 2011;8:446-9.

36. Flicek KT, Hara AK, Silva AC, et al. Reducing the radiation dose for CT colonography using adaptive statistical iterative reconstruction: A pilot study. AJR Am J Roentgenol. 2010;195:126-31.

37. Heresbach D, Djabbari M, Riou F, et al. Accuracy of computed tomographic colonography in a nationwide multicentre trial, and its relation to radiologist expertise. Gut. 2011;60:658-65.

38. Hara AK, Blevins M, Chen MH, et al. ACRIN CT Colonography Trial: Does Reader's Preference for Primary Two-dimensional versus Primary Three-dimensional Interpretation Affect Performance? Radiology 2011;259:435-41.

39. Johnson CD, Manduca A, Fletcher JG, et al. Noncathartic CT colonography with stool tagging: performance with and without electronic stool subtraction. AJR Am J Roentgenol 2008;190:361-6.

40. Zalis ME, Blake MA, Cai W, et al. Diagnostic accuracy of laxative-free computed tomographic colonography for detection of adenomatous polyps in asymptomatic adults: a prospective evaluation. Ann Intern Med. $2012 ; 156: 692-702$.

41. Halligan S, Mallett S, Altman DG, et al. Incremental benefit of computer-aided detection when used as a second and concurrent reader of CT colonographic data: multiobserver study. Radiology 2011;258:469-76

42. Mang T, Bogoni L, Salganicoff M, et al. Computer-aided detection of colorectal polyps in CT colonography with and without fecal tagging: a stand-alone evaluation. Invest Radiol. 2012 Feb;47(2):99-108.

43. Zalis ME, Barish MA, Choi JR, et al. For the Working Group on Virtual Colonoscopy. CT colonography reporting and data system: a consensus proposal. Radiology 2005;236:3-9.

44. Yee J, Kumar NN, Godara S et al. Extracolonic abnormalities discovered incidentally at CT colonography in a male population. Radiology 2005; 236, 519-526.

45. Yee J, Sadda S, Aslam R, Yeh B. Extracolonic Findings at CT colonography. Gastrointest Endoscopy Clin N Am 2010; 20: 305-322.

46. Macari M, Nevsky G, Bonavita J, et al. CT colonography in senior versus nonsenior patients: extracolonic findings, recommendations for additional imaging, and polyp prevalence. Radiology. 2011;259:767-74. 
47. Sutherland T, Coyle E, Lui B, et al. Extracolonic findings at CT colonography: a review of 258 consecutive cases. J Med Imaging Radiat Oncol. 2011;55:149-52.

48. Xiong T, Richardson M, Woodroffe R, et al. Incidental lesions found on CT colonography:their nature and frequency. Br J Radiol 2005;78:22-9.

49. Veerappan GR, Ally MR, Choi JH, Pak JS, Maydonovitch C, Wong RK. Extracolonic findings on CT colonography increases yield of colorectal cancer screening. AJR Am J Roentgenol 2010;195:677-86.

50. Pickardt PJ, Kim DH, Meiners RJ, et al. Colorectal and Extracolonic Cancers Detected at Screening CT colonography in 10286 Asymptomatic Adults. Radiology 2010;255:83-88.

51. Ichikawa T, Kawada S, Hirata S, et al. Initial experience with computed tomographic colonography applied for noncolorectal cancerous conditions. Jpn J Radiol. 2011;29:386-93.

52. Berland LL, Silverman SG, Gore RM, Mayo-Smith WW, et al. Managing incidental findings on abdominal CT: white paper of the ACR incidental findings committee. J Am Coll Radiol 2010;7:754-48. 